\section{Brain reflections}

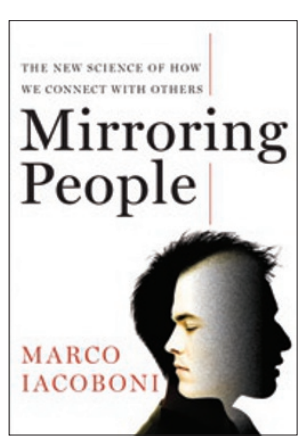

\section{Mirroring People \\ by Marco lacoboni}

Farrar, Straus \& Giroux, 2008

320 pp, hardcover, \$25

ISBN 0374210179

\section{Reviewed by Ullrich Wagner \& Patrik Vuilleumier}

Sometimes scientific progress emerges from accidental findings. In the 1980s a group of Italian neuroscientists in the laboratory of Giacomo Rizzolatti, in Parma, implanted microelectrodes into motor areas of monkey brains to record single-cell activity related to movements performed by the animals. They found that some of the neurons were highly specialized for certain actions. For example, some neurons fired only when the monkey grasped a big object such as an apple, but not when it grasped a small object such as a peanut. This was already an interesting feature, but then the researchers found another totally unexpected property: the same neurons that were activated when the monkey grasped an apple also fired when the monkey merely observed another monkey (or the experimenter) grasping an apple, without performing any overt movement itself. Because it seemed that the monkey internally imitated, or 'mirrors', the action that it observed in others, the term "mirror neurons" was coined for these cells. With this discovery, a new research direction was born, which ever since has provided fascinating insights into a variety of behaviors in both monkeys and humans.

In his new book Mirroring People, Marco Iacoboni provides a comprehensive account of what this research has revealed so far. Iacoboni, a neurologist at the University of California Los Angeles, is a leading expert on this topic, whose research group has performed many of the human brain imaging studies in the field and who-having Italian roots himself-has tight links with Rizzolatti's group in Parma. He tells the story systematically, beginning with the initial discovery of mirror neurons in monkeys and turning subsequently to human research, with each chapter focusing on a new empirical question and the relevant results.

What makes this book particularly interesting is that it does not simply summarize the current state of the research itself; the author also uses the existing findings to draw more general conclusions about human nature. According to Iacoboni, the data are pertinent to essential philosophical, psychological and even political questions, and he convincingly argues that the mirror neuron system can be a

Ullrich Wagner and Patrik Vuilleumier are at the Department of Neuroscience, University Medical School, at the Center for Neuroscience, and at the Swiss Center for Affective Sciences, University of Geneva, Geneva, Switzerland. e-mail: ullrich.wagner@unige.ch key to answering many such questions. For example, mirror neurons may lend support to psychological theories that regard imitation as the primary mechanism underlying our understanding of the inner mental states of other people. If mirror neurons represent an immediate, covert imitation of what others do, this could be a way in which we directly understand what they feel and what they intend. Mirror neurons might also address the philosophical problem of intersubjectivity, the sharing of meaning between people, as they could provide us with a direct representation of what others mean. Thus, in line with existential phenomenologists such as Merleau-Ponty or Wittgenstein, we immediately perceive emotions, not muscle movements, when we observe a face. The critical point is that the understanding supported by mirror neurons is fast and intuitive, and needs no further inferential processing. The brain is literally reflecting the other, not reflecting on the other. Or, as Iacoboni says, "the mirror neuron system seems to project internally [...] other people into our own brains" (p.260).

This is in fact the deeper central theme of the book: humans are socially defined in that the self and the other are closely interdependent, connected by their mirror neurons (hence the subtitle: "The new science of how we connect with others"). Mirror neurons allow us not only to understand others, but also to empathize or even identify with them. They are the foundation of most human social capabilities. Following his interdisciplinary interest, Iacoboni applies this idea to a variety of different areas including, among others, language acquisition, autism, media violence and politics. For the more broadly interested reader, this journey through different domains provides a fascinating perspective on theoretical discussions outside neuroscience.

However, not every one of Iacoboni's assumptions about a critical role for mirror neurons is equally convincing. His obvious enthusiasm about this research sometimes leads him to overinterpretations that are difficult to comprehend, particularly in the field he calls "neuropolitics." For example, during the US presidential election year of 2004, Iacoboni's group found that Democratic and Republican partisans who watched their own candidate, as compared to other candidates, showed relative activation of the middle orbitofrontal cortex (a region containing what Iacoboni calls "super mirror neurons") when scanned in spring, but not when scanned in late summer. Iacoboni interprets this change as a reduction of identification with their own candidate because of negative information accumulated during the political campaigns. Of course, many other factors not relating to mirror processes could also account for such a change. But Iacoboni, as one of the driving forces in this field of research, seems inclined to see mirror neurons at work even in cases where the evidence for their effects is quite indirect. The reader who expects to get an expert's view of certain shortcomings or limitations of mirror neuron research will search in vain for it in this book.

Still, Mirroring People is highly recommendable both to neuroscientists (particularly those working in social neuroscience) and to a broader audience. Its clear structure and simple language, avoiding technical terms, make it easy to understand even for nonexperts. The concept of mirror neurons is a popular topic today, and many scientifically interested people will have heard of it in one way or another. This book gives them the opportunity to learn more about the specific results and ideas that lie behind this concept. 